\title{
Possibility of using the beet dyes as a laser gain medium
}

\author{
Abdelaziz Hagar Abdelrahman ${ }^{1,2^{*}}$, Malik A. Abdelrahman ${ }^{3,4}$, Mohammed Khaled Elbadawy ${ }^{3}$ \\ ${ }^{1}$ Laser Institute, Sudan University for Science and Technology (SUST), Khartoum, Sudan; \\ *Corresponding Author: abdelazeez@hotmail.com \\ ${ }^{2}$ Physics Department, Collage of Science \& Arts, Muznab, Qassim University, Qassim, KSA \\ ${ }^{3}$ Chemistry Department (SUST), Khartoum, Sudan \\ ${ }^{4}$ Chemistry Department, Collage of Applied Medical Sciences, (TURUBA), Taif University, Taif, KSA
}

Received 27 July 2013; revised 27 August 2013; accepted 4 September 2013

Copyright (C) 2013 Abdelaziz Hagar Abdelrahman et al. This is an open access article distributed under the Creative Commons Attribution License, which permits unrestricted use, distribution, and reproduction in any medium, provided the original work is properly cited.

\begin{abstract}
Nowadays the dye lasers play as an important tool and are used in many applications including spectroscopy, medicine and dermatology. This research was carried out to study the possibility of using the beet dyes as a laser gain medium. The fluorescence quantum yield was determined by the comparative method with rodamine $b$ as an organic dye standard. The value of the fluorescence quantum yield was found about $(0.14)$ and the fluorescence quantum yield was developed until reaching about $(0.323)$. The increasing of fluorescence quantum yield of dye solution as a result of increasing the viscosity of solvent was observed clearly. The study concluded that the beet dyes are so sensitive to fluorescence and it is very suitable to be used as a laser gain medium.
\end{abstract}

Keywords: Beets; Lasing; Solvents; Quantum Yield

\section{INTRODUCTION}

From the mid 60 s, dye lasers have been attractive sources of coherent tunable radiation because of their unique operational flexibility [1]. Light amplification by stimulated emission of radiation, that is lasers, has become an important tool in chemistry and many sciences. A dye laser can be defined as a laser utilizing dye [2]. Some of the most popular and heavily investigated are the rhodamines and coumarins. Dye lasers have many advantages over their liquid counterparts. They are nontoxic, nonflammable, and can be engineered to be rugged and compacted by eliminating the dye flow hardware that is necessary in a liquid system. Liquid dye lasers rely on dye flow through the laser cavity to avoid thermal problems and the buildup of photo degradated dye molecules which decrease the lasing efficiency [3].

The knowledge of fluorescence quantum efficiency of organic dyes is important in selecting efficient dye laser media [4]. Organic dyes dissolved in suitable liquid solvent have found wide application in laser technology serving as an active gain medium for generation of coherent tunable radiation [5].

A dye laser is a laser which uses an organicdye as the lasing medium, usually as a liquidsolution. For effective performance, dye molecules should have strong absorption at excitation wavelength and minimal absorption at lasing wavelength. Applications of lasers are wide and varied today. They are found in communication techniques, microsurgery and medicine [6].

\section{QUANTUM YIELD}

The quantum yield of a radiation-induced process is the number of times that a defined event occurs per photon absorbed by the system. Thus, the quantum yield is a measure of the efficiency with which absorbed light produces some effect [7].

Quantum yield is essentially the emission efficiency of a given fluoro chrome. Since not all photons are absorbed productively, the typical quantum yield will be less than 1 [8].

The fluorescence quantum yield is defined as the ratio of the number of photons emitted to the number of photons absorbed or we can written as .[9].

$$
Q Y=\frac{\text { number of photons emitted }}{\text { number of photons absorbed }}
$$

In comparative method, the same for different sample solutions compared under identical conditions of excita- 
tion [9]. We may re-write Equation (2.1) as:

$$
Q Y=\frac{\alpha(\text { number of photons detected })}{\beta(\text { fraction of light absorbed }(F))}
$$

where $\alpha$ and $\beta$ are constants related to the fraction of emitted light entering the light collection optics of the fluorimeter and the intensity of the excitation source at the excitation wavelength, respectively. In this equation, the number of photons detected is the integrated area under the corrected fluorescence spectrum and the fraction of light absorbed $(F)$ is obtained from the absorbance at the excitation wavelength by;

$$
F=1-10^{-D}
$$

where $(D)$ defined as an optical density of a medium is the measure of its transmittance for a radiation of particular wavelength. The relationship between the optical density and transmittance $(T)$ is an inverse, which expressed as [10].

$$
D=\log _{10}(1 / T)
$$

Hence, for sample $X$, we can write:

$$
Q Y_{x}=\frac{\propto A_{x}}{\beta F_{x}}
$$

where $A_{x}$ is the integrated area under the corrected fluorescence spectrum. For a "standard" material, $S$, for which the quantum yield of fluorescence is already known, we can similarly write:

$$
Q Y_{s}=\frac{\propto A_{s}}{\beta F_{s}}
$$

Comparative measurements on solutions of sample $X$ and a suitable standard, $S$, can then be used to estimate the fluorescence quantum yield of $X$ by dividing Equation (2.4) by Equation (2.5):

$$
\frac{Q Y_{x}}{Q Y_{s}}=\frac{A_{x}}{A_{s}} \frac{F_{s}}{F_{x}}
$$

where upon the unknown constants $\boldsymbol{\alpha}$ and $\boldsymbol{\beta}$ cancel out.

Hence:

$$
Q Y_{x}=Q Y_{s}\left(\frac{A_{x}}{A_{s}} \frac{F_{s}}{F_{x}}\right)
$$

where all terms on the right hand side of Equation (2.7) are known. It is assumes that measurements on solutions of $X$ and $S$ are made under identical conditions of excitation wavelength and aperture settings. If different solvents are used for $X$ and $S$, then Equation (2.7) should be further modified to allow for the effect of refractive index on the relative amount of fluorescence collected by the fluorimeter detection optics. The final equation for estimation of fluorescence quantum yields by the Comparative Method then becomes:

$$
Q Y_{x}=Q Y_{s}\left(\frac{A_{x}}{A_{s}} \frac{F_{s}}{F_{x}}\right)\left(\frac{n_{x}}{n_{s}}\right)^{2}
$$

where the $n_{s}$ and $n_{x}$ are the refractive indices of the solvents used for the two solutions fluorescence spectra are always fully corrected using the installed correction function of the Jobin Yvon Fluorolog spectro fluorimeter. This function corrects for the variation with wavelength of the sensitivity of the photomultiplier and the spectral characteristics of the detection optics (gratings, mirrors, etc.) [9].

In practice at nanotechnologies, solutions of quantum yield material are compared with solutions of organic fluorophores of known quantum yield under the same conditions of excitation. Absorbance's of all solutions are adjusted so as to be preferably in the range $(0.02-0.07)$ and not greater than ca. 0.1 at the excitation wavelength. If possible, it is best to arrange for the absorbencies of the sample and standard solutions to be the same (or nearly the same) at the exciting wavelength. Organic controls that have been used are as shown in Table 1 [9].

\section{CHOICE OF SOLVENT}

Prepared laser dye solutions usually contain very small quantities of dye. Typical dye concentrations are $10^{-2}$ to $10^{-5}$ molar. For this reason, the solvent in which the dye is dissolved plays an important role when defining physical properties and potential hazards.

Table 1. Quantum yields determined by this method are reliable to about $\pm 10 \%$.

\begin{tabular}{ccccc}
\hline Standard & Solvent & QY & Fluorescence max (nm) & Useful excitation range (nm) \\
\hline 9.10-diphenylanthracene & Ethanol & 0.90 & $406-4.27$ & $320-370$ \\
Coumarin 1 & Ethanol & 0.70 & 450 & $320-370$ \\
Rhodamine 123 & Ethanol & 0.96 & 535 & $440-490$ \\
Rhodamine 6G & Ethanol & 0.95 & 560 & $450-500$ \\
Sulphorhodamine 101 & Ethanol & 0.95 & 600 & $500-550$ \\
\hline
\end{tabular}


Lasing wavelength and energy are very sensitive to the choice of solvent. Most laser dyes are polar molecules, and excitation into their lowest-lying singlet state is accompanied by an increase in the dipole moment. Accordingly, solvent polarity plays an important role in shifting the lasing wavelength. In a majority of circumstances, increasing solvent polarity will shift the gain curve toward longer wavelength. In the case of more polar dyes, the shift can be as high as $20-60 \mathrm{~nm}$.

The output power of dye lasers is strongly dependent on the purity of the solvent. Impurities and additives may strongly affect upper state lifetime of the dye or may catalyse photochemical reactions. Therefore, for best results, only high quality solvents are to be recommended [11].

With the exception of water, all solvents should be considered hazardous. In many instances, the solvent in which the dye is dissolved plays a major role in the hazard presented by the final solution.

Water is called the universal solvent because more substances dissolve in water than in any other chemical. This has to do with the polarity of each water molecule. The hydrogen side of each water $\left(\mathrm{H}_{2} \mathrm{O}\right)$ molecule carries a slight positive electric charge, while the oxygen side carries a slight negative electric charge. This helps water dissociate ionic compounds into their positive and negative ions. The positive part of an ionic compound is attracted to the oxygen side of water while the negative portion of the compound is attracted to the hydrogen side of water.

These hazards must be addressed carefully in dye handling and solution preparation. Nearly all solvents are highly flammable. Therefore, a small fire extinguisher should be installed near the laser in a readily accessible and unobstructed area.

A particular fire hazard that is not commonly known occurs with nonpolar and, hence, nonconductive solvents. If these solvents are circulated at a high speed through plastic tubings, the pump unit acts as a van de Graff generator, producing up to $100 \mathrm{kV}$, and sparks may pierce the tubing and ignite the solvent. The dye selectors use grounding wires inside the plastic tubings to eliminate these problems. However, when using such solvents, check first for static electricity before opening the reservoir. Static electricity is present when hair on the back of your hand or forearm is attracted to the plastic tubing. do not circulate dye solutions made with such solvents for more than a minute, unless the cuvette has been placed into the crate and is grounded [12].

\section{BEET ROOTS AND BEET DYES}

The beet (Beta vulgaris) is a plant in the amaranth family. It is best known in its numerous cultivated varieties, the most well-known of which is probably the red root vegetable known as the beetroot or garden beet. However, other cultivated varieties include the leaf vegetables chard and spinach beet, as well as the root vegetables sugar beet, which is important in the production of table sugar, and mangel-wurzel, which is a fodder crop. Three subspecies are typically recognized. All cultivated varieties fall into the subspeciesBeta vulgaris subsp. vulgaris, while Beta vulgaris subsp. maritima, commonly known as the sea beet, is the wild ancestor of these and is found throughout the Mediterranean, the Atlantic coast of Europe, the Near East, and India. A second wild subspecies, Beta vulgaris subsp. adanensis, occurs from Greece to Syria.

Betacyanins $(\mathrm{B} c)$ is extracted dyes from beetroot or beet red (Beta vulgaris). The two hydro-soluble compounds: betacyanin $(\mathrm{Bc})$ and betaxanthins $(\mathrm{Bx})$, which present absorption bands at 540 and $480 \mathrm{~nm}$, respectively are obtained from aqueous extraction of beet red [13] as seen in Figure 1.

\section{METHODOLOGY}

\subsection{Sample Preparation}

The beet samples were collected from local markets in Khartoum state and the dye solution was extracted by water and the extracted was separated by filtration. Then the ability of dyes solution to work as a laser gain medium has been testified.

The dye solution was extracted by $0.1 \%$ aqueous TFA (Tetra Fluoro Acetic acid).

Sample one: $1 \mathrm{ml}$ of dye 0.1 solution was added to 100 $\mathrm{ml}$ of water (the absorbance of sample was adjusted to be less than 0.1 au. Four different concentrations were prepared by water dilution in the range of $(0.1-0.01$ au abs).

Sample two: $1 \mathrm{ml}$ of dye solution was added to $100 \mathrm{ml}$ of glycerin-water (1:10) solution. Four different concentrations were prepared by dilution in the range of $(0.1$ $0.01 \mathrm{au}$ abs).

Sample three: $1 \mathrm{ml}$ of dye solution was added to 100 $\mathrm{ml}$ of glycerin-water $(2: 10)$ solution. Four different con-
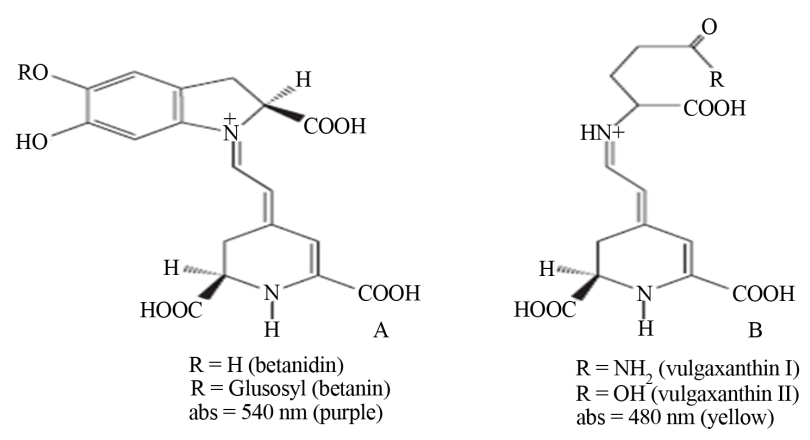

Figure 1. Structure of the main beetroot dyes. (A) betacyanins $(\mathrm{Bc})$ also known as betanin (with sugar moiety) and betanidine without sugar and $(\mathrm{B})$ betaxanthins $(\mathrm{Bx})$. 
centrations were prepared by dilution in the range of $(0.1-$ $0.01 \mathrm{au}$ abs).

Sample four: $1 \mathrm{ml}$ of dye solution was added to $100 \mathrm{ml}$ of glycerin-water (4:10) solution. Four different concentrations were prepared by dilution in range of $(0.1-0.01$ au abs).

\subsection{Measurement Procedure}

First step the absorbance at $538 \mathrm{~nm}$ for solution of sample one was recorded, second one the fluorescence spectrum of the same solution was recorded in $10 \mathrm{~mm}$ fluorescence cuvette. Step 1 and 2 were repeated for four solutions with increasing concentration of sample one.

Ostwald Device was used to determine the viscosities of samples solvents.

Refractive indices and densities were determined by using the mettler Toledo DR40 refractometer. Then the fluorescence quantum yields of the solvents were determined by using the value of the refractive indexes which given above.

\section{RESULT AND DISSECTION}

Table 2 shows relationship between the maximum wavelength of light absorption $\left(\lambda_{\max }\right)$ and beet dyes on different $\mathrm{pH}$ was observed. The $\lambda_{\max }$ of light absorption is shifted as a result of the $\mathrm{pH}$ varying and the dye solution showed instability at $\mathrm{pH}$ more than 8

Figure 2 showed four peaks and the HPLC fluorescence was running in $530 \mathrm{~nm}$ excitation wave length and the emission wave length at $630 \mathrm{~nm}$ the result also showed four strong peaks. One of the four components displayed high emission peak this might mean that the dye solution should have a significant fluorescence quantum yield.

Fluorescence of a molecule depends on the structure and environment of the molecule such as interaction with solvent and other dissolved compounds in the matrix, the $\mathrm{pH}$ and concentration of the fluorescing species. UncalCalib $\backslash$ beet root mix 2 shown in Table 3.
The dye solutions of various concentrations were pumped by a He-Ne flash lamp laser and the scatter light was collected at $90^{\circ}$ from the cuvette but unfortunately the source of laser emission is weak at green area which is absorbed by the dye solution, the results showed fluorescence $\lambda_{\max }$ that differ from fluorescence $\lambda_{\max }$ of $\mathrm{He}-\mathrm{Ne}$ flash lamp laser $(632.8 \mathrm{~nm})$ that means the dye solution should have a significant fluorescence quantum yield.

\section{CALCULATION OF FLUORESCENCE QUANTUM YIELD}

To get fluorescence efficiency of various concentrations of beet dyes a He-Ne flash lamp laser pumped them to obtain fluorescence spectra peaks. Fluorescence spectra peaks of various concentrations of beet dyes which are pumped by a He-Ne flash lamp laser.

\subsection{Density and Refractive Indices of Solvents Samples Were Determined as in Table 4}

\subsection{Fluorescence Quantum Yields}

To calculate the fluorescence quantum yields for beet dyes on different solvents, one can use the comparative method by this equation;

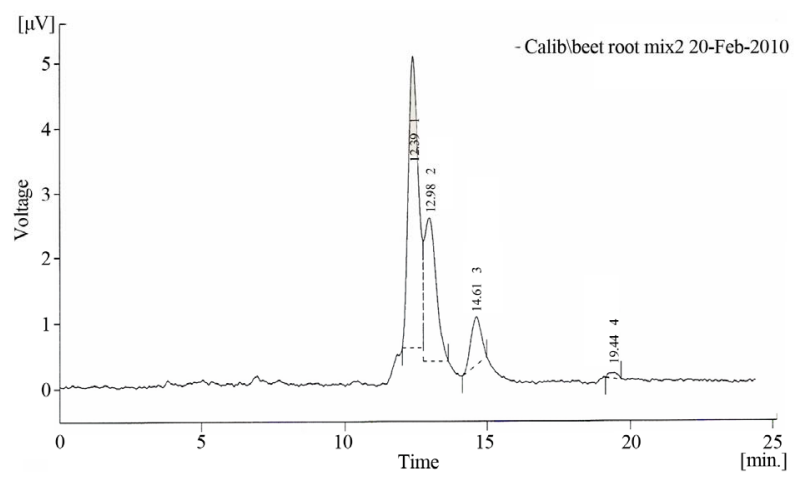

Figure 2. HPLC-fluorescence for beet dyes solution.

Table 2. $\lambda_{\max }$ depend on $\mathrm{pH}$ of solvent.

\begin{tabular}{|c|c|c|c|c|c|c|c|c|c|c|}
\hline $\mathrm{pH}$ & 3.15 & 4.07 & 5.15 & 6.30 & 7.19 & 8.24 & 9.03 & 10.15 & 11.00 & 12.00 \\
\hline$\lambda_{\max }(\mathrm{nm})$ & 485 & 485 & 475 & 475 & 480 & ------ & ------ & 395 & ------ & ------ \\
\hline
\end{tabular}

Table 3. Shows (Uncal-Calib \beet root mix2).

\begin{tabular}{ccccccc}
\hline & Reten. Time $[\mathrm{min}]$ & Area [mV.s] & Height [mV] & Area [\%] & Height [\%] & W05 [min] \\
\hline 1 & 12.388 & 22.533 & 0.895 & 57.3 & 59.7 & 0.42 \\
2 & 12.984 & 12.664 & 0.441 & 32.2 & 29.4 & 0.47 \\
3 & 14.612 & 3.703 & 0.147 & 9.4 & 9.8 & 0.43 \\
4 & 19.436 & 0.398 & 0.018 & 1 & 1.2 & 0.43 \\
& Total & 39.298 & 1.5 & 100 & & 100 \\
\hline
\end{tabular}


Table 4. Densityand refractive indices of solvents of beet dyes solutions.

\begin{tabular}{ccc}
\hline No of sample & Density $\left(\mathrm{g} / \mathrm{cm}^{3}\right)$ at $23^{\circ} \mathrm{C}$ & Refractive indices \\
\hline 1 & 0.9979 & 1.334 \\
2 & 1.0229 & 1.342 \\
3 & 1.0563 & 1.361 \\
4 & 1.0890 & 1.380 \\
\hline
\end{tabular}

$$
\Phi_{X}=\Phi_{S T}\left(\frac{\operatorname{Grand}_{X}}{\operatorname{Grand}_{S T}}\right)\left(\frac{\eta_{X}}{\eta_{S T}}\right)
$$

where, $\Phi_{X}, \Phi_{S T}, \eta_{x}, \eta_{S T}$ stand for fluorescence quantum field of sample, fluorescence quantum field of standard, refractive index of sample solventand refractive index of standard solvent respectively. The value of $\Phi_{X}$ of four samples were obtain, as shown in Table 5.

Calculation of the viscosity of solvents:

The calculation of the viscosity of solvents were shown in Table 6.

The relationship between fluorescence quantum yield and viscosity of solvent isrepresented as in the Figure 3.

The result gave a logarithmic function. The values of Log Fluorescence quantum yieldand Log Viscosity of solvent were presented in Table 7.

The relation of Log Fluorescence quantum yield and Log Viscosity of solvent were presented in Figure 4.

The relationship between fluorescence quantum yield $(\Phi)$ and the viscosity $(\lambda)$ of the solvent has been derived analytically and experimentally and is known as the Forster-Hoffmann equation $(\log \Phi=C+x \log \lambda)$ where $\mathrm{C}$ is a temperature-dependent constant and $\mathrm{x}$ is a dyedependent constant [14].

\section{CONCLUSIONS}

The fluorescence quantum yields of beet dyes solutions were determined by the comparative method with rhodamine $b$ as standard. The fluorescence quantum yield was developed by increasing the viscosity of solvents as shown in Tables 6 and 7.

The result in Table 4 established that the beet dyes solution has a high fluorescence quantum yield to act as a laser gain medium.

Figure 2 shows four peaks, one of these components displayed high emission, and this means that the dye solution should have a significant fluorescence quantum yield, which leads the solvent to act as gain medium.

The increasing of fluorescence quantum yield value as a result of increasing the viscosity of solvent means that the dye behaves like intramolecular charge transfer (ICT) compounds because of the nitrogen atom attached the $\pi$-conjugation system [14].
Table 5. The values of fluorescence quantum yields for beet dyes on different solvents.

\begin{tabular}{ccccccc}
\hline$\Phi_{x}$ & $\eta_{x}$ & $\eta_{S T}$ & $\Phi_{S T}$ & $\operatorname{Grad}_{S T}$ & $\operatorname{Grad}_{x}$ & No Sample \\
\hline 0.114 & 1.344 & 1.334 & 0.31 & 899 & 313.5 & 1 \\
0.169 & 1.342 & 1.334 & 0.31 & 899 & 483 & 2 \\
0.200 & 1.361 & 1.334 & 0.31 & 899 & 577.7 & 3 \\
0.323 & 1.380 & 1.334 & 0.31 & 899 & 874.2 & 4 \\
\hline
\end{tabular}

Table 6. Viscosity values of solvents.

\begin{tabular}{ccc}
\hline Viscosity (cent-poise) & Solvent (ml:ml) & No Sample \\
\hline 0.9325 & Water & 1 \\
2.1092 & Water-glycerin $(23: 2)$ & 2 \\
3.7860 & Water-glycerin (20:5) & 3 \\
7.6730 & Water-glycerin (17:8) & 4 \\
\hline
\end{tabular}

Table 7. Logarithmic data.

\begin{tabular}{cc}
\hline Log Viscosity of solvent & Log Fluorescence quantum yield \\
\hline-0.0303 & -0.943 \\
0.3228 & -0.772 \\
0.5782 & -0.699 \\
0.8900 & -0.491 \\
\hline
\end{tabular}

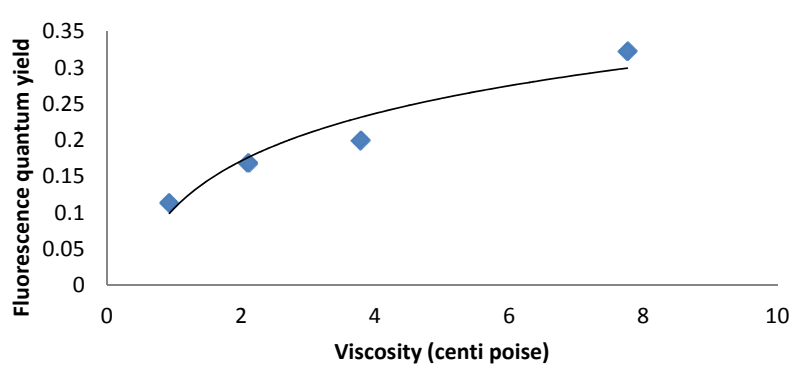

Figure 3. Relationship between fluorescence quantum yield and viscosity of solvent.

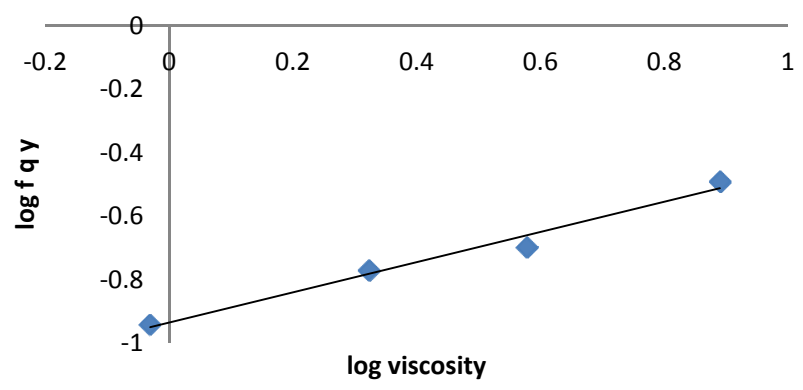

Figure 4. Function linearization.

On the basis of the results presented in this paper, it can initially be concluded that lasing in beet dyes solution is 
possible. This is in agreement with the results of fluorescence quantum yield values of beet dyes solutions.

The measurements showed that the lasing wavelength depends on the $\mathrm{pH}$ of the solvent and also the efficiency of fluorescence depends on the viscosity of solvent.

\section{REFERENCES}

[1] Namoori, V.P.N. and Vallabhan, C.P.G. (2002) Studies on fluorescence efficiency and photodegradation of rhodamine 6G doped PMMA using a dual beam thermal lens technique, Laser Chemistry, 20, pp. 99-110.

[2] Steen, M. (year) Laser materials processing.

[3] Sathy, P., Rejiphilip, V.P., Nampoori, N. and Vallabhan, C.P.G. (1990) Fluorescence quantum yield of rhodamine 6G using pulsedpbotoacoustictecbnique, Pramana Journal of Physics, 34, 585-590.

[4] Shankarling, G.S. and Jarag, K.J. (2010) Laser dyes. Institute of Chemical Technology, Matunga Mumbai, 400 019.

[5] Schaefer F.P. (1977) Dye Lasers. 2nd Edition, Verlag, New York.

[6] Schaefer, F.P. (1973/1977) Structure and properties of laser dyes. In: Drexhage, K.H., Ed., Dye Laser Vol. 1 Topics in Applied Physics, Springer Verlag, Hamburg.

[7] Maeda, M. (1984) Laser dyes, properties of organic com- pounds for dye lasers. Academic Press, Inc., Orlando, 1.

[8] Drexhage, K.H. (1990) Structure and properties of laser dyes in topics in applied physics. Springer-Verlag, Berlin, 21-22,167-169.

[9] Ahluwalia, G.S. (2008) Cosmetic application of laser and light-based system. William Andrew, 101.

[10] Tian, K., Thomson, K.A., Liu, F.S., Snelling, D.R., Smallwood, G.J. and Wang, D.S. (2004) Determination of the morphology of soot aggregates using the refractive optical density method for the analysis of TEM images. In: Daintith, J., Ed., Combustion and Flame 144-(2006) -782-791, Dictionary of Chemistry, Oxford university Press, Elsevier, 475.

[11] Tuchin, V.V. (2007) Tissue optics: Light scattering method and instruments for medical diagnosis. SPIE Press, 642.

[12] (2008) Protocol for Fluorescence Quantum Yield Determination Employed by Nanoco Technologies Ltd.

[13] Bonacin, J.A., Engelmann, F.M., Severino, D., Toma, H.E. and Baptista, M.S. (2009) Singlet oxygen quantum yields $(\varphi \Delta)$ in water using beetroot extract and an array of LEDs. Journal of the Brazilian Chemical Society, 20, 31-36.

[14] Haidekker and Theodorakis (2010) Intrinsic and extrinsic temperature-dependency of viscosity-sensitive fluorescent molecular rotorsvolume. Journal of Biological Engineering, 4, 4-11. 